\title{
Spectral splittings in the Conley index theory
}

\author{
Piotr Bartłomiejczyk
}

To Professor Kazimierz Geba on his 80th birthday

\begin{abstract}
We introduce spectral splittings and spectral graphs. We explain the relation between spectral splittings, graphs and sequences.
\end{abstract}

Mathematics Subject Classification. Primary 55T05; Secondary 37B30.

Keywords. Spectral sequence, Morse decomposition, connection matrix.

\section{Introduction}

One of the main ideas behind the Conley index theory is to apply the tools from algebraic topology in studying dynamical systems, especially the structure of invariant sets (see $[5,6,10,14]$ ). This approach, motivated by Morse theory, focuses on decomposing isolated invariant sets into invariant subsets (Morse sets) and connecting orbits between them. This structure is called a Morse decomposition of an isolated invariant set. A filtration of index pairs associated with a Morse decomposition can be used to find connections between different Morse sets. The principal tools for this purpose are connection matrices (see $[3,4,9,13])$, connection graphs (see $[2,8]$ ) and spectral sequences (see $[1,4,7]$ ).

The main goal of this paper is to explain and clarify the basic relations between connection matrices (generalized here to spectral splittings), connection graphs (called here spectral graphs) and spectral sequences. Although the comparison is done mainly on algebraic level, we hope that it sheds some new light on important aspects of the Conley index theory. This paper is mainly intended as a brief survey summarizing results from $[1,2,3,4]$.

The organization of the paper is as follows. Section 2 contains some preliminaries. In Section 3 we introduce spectral splittings and study their basic properties. In Section 4 we briefly explain the relation between spectral splittings and connection matrices. Section 5 contains a brief description of spectral graphs. Finally, in Section 6 we use spectral splittings and graphs 
to study the structure of the Morse decomposition of a flow on a compact metric space.

\section{Preliminaries}

Recall that a filtered vector space is a vector space $A$ equipped with a finite increasing filtration, that is, a sequence $\left\{A^{p}\right\}_{0}^{n}$ of subspaces of $A$ such that

$$
0=A^{0} \subset A^{1} \subset \cdots \subset A^{n}=A .
$$

We will use the following convenient notation:

$$
\begin{array}{ll}
A^{p}=A & \text { for } p \geq n, \\
A^{p}=0 & \text { for } p \leq 0 .
\end{array}
$$

Definition 2.1. A filtered differential vector space ( $f$ - $d$ space for short) is a filtered vector space $A$ together with an endomorphism $d$ such that $d^{2}=0$ and $d$ preserves the filtration, i.e., $d A^{p} \subset A^{p}$.

Remark 2.2. The main advantage of using filtered differential vector spaces instead od filtered differential modules is that we can apply standard methods of linear algebra, but this approach has also the disadvantage that we obtain homology with coefficients in a field, which is blind to torsion.

Observe that we have two natural finite filtrations associated with the f-d space. Namely,

$$
\begin{aligned}
& 0=A^{0} \subset A^{1} \subset \cdots \subset A^{n}=A, \\
& 0=d A^{0} \subset d A^{1} \subset \cdots \subset d A \subset d^{-1} 0 \subset d^{-1} A^{1} \subset \cdots \subset d^{-1} A^{n}=A .
\end{aligned}
$$

Let us briefly recall the definition of the spectral sequences of the $\mathrm{f}-\mathrm{d}$ space (see [15] for more details). We introduce the following notation. Let

$$
\begin{aligned}
& Z_{p}^{r}:=A^{p} \cap d^{-1} A^{p-r}, \\
& B_{p}^{r}:=A^{p} \cap d A^{p+r}
\end{aligned}
$$

for any $r \in \mathbb{Z}^{+}$and $p \in \mathbb{Z}$. Since, as is easy to see, $Z_{p-1}^{r-1} \subset Z_{p}^{r}$ and $B_{p}^{r-1} \subset Z_{p}^{r}$, the quotient vector space

$$
E_{p}^{r}:=\frac{Z_{p}^{r}}{Z_{p-1}^{r-1}+B_{p}^{r-1}}
$$

is well defined. Moreover, since the differential $d$ induces homomorphisms

$$
Z_{p}^{r} \rightarrow Z_{p-r}^{r}, \quad Z_{p-1}^{r-1}+B_{p}^{r-1} \rightarrow Z_{p-r-1}^{r-1}+B_{p-r}^{r-1},
$$

it also induces the homomorphism of quotient vector spaces, which we will denote by $d_{p}^{r}$ :

$$
d_{p}^{r}: E_{p}^{r} \rightarrow E_{p-r}^{r}
$$

Observe that $d_{p}^{r}([z])=[d z]$, where $[\cdot]$ denotes the respective equivalence class. From this we obtain $d_{p-r}^{r} d_{p}^{r}[z]=[d d z]=0$ and so $d_{p-r}^{r} d_{p}^{r}=0$. For a 
fixed $r$ homomorphisms $d_{p}^{r}$ induce the homomorphism

$$
d^{r}: \bigoplus_{p} E_{p}^{r} \rightarrow \bigoplus_{p} E_{p}^{r} .
$$

Hence $d^{r}$ is a differential of a vector space $E^{r}=\bigoplus_{p} E_{p}^{r}$.

Definition 2.3. A sequence of vector spaces and differentials $\left\{E^{r}, d^{r}\right\}_{r=1}^{\infty}$ is called the spectral sequence of the $f$-d space $A$.

Remark 2.4. It is easily seen that if the filtration of the $\mathrm{f}$-d space $A$ has length $n$, then the spectral sequence stabilizes at the $n$th term; i.e., $E^{r} \simeq$ $E^{r+1}$ for $r \geq n$.

In the next section we will make use of the following two easy-to-check facts from linear algebra.

Lemma 2.5. Assume that

- $L: V \rightarrow V$ is linear,

- $V=U \oplus A$ with $\operatorname{ker} L \subset U$,

- $L V=L U \oplus B$ and $L A \subset B$,

then $L: A \rightarrow B$ is an isomorphism.

Lemma 2.6. If $L: V \rightarrow V$ is linear, $U$ is a linear subspace of $V$ and $L V=$ $L U \oplus B$, then there is a linear subspace $A$ of $V$ such that $V=U \oplus A$ and $L A \subset B$.

\section{Spectral splittings of filtered differential vector spaces}

We introduce unified notation for elements of the filtration (2.2). Namely, let

$$
D^{m}= \begin{cases}d A^{m} & \text { for } m \leq n, \\ d^{-1} A^{m-n-1} & \text { for } m>n .\end{cases}
$$

Definition 3.1. A spectral splitting of the $\mathrm{f}-\mathrm{d}$ space $A$ is a sequence

$$
\left\{A_{p, q}\right\} \begin{gathered}
1 \leq p \leq n \\
0 \leq q \leq n+1
\end{gathered}
$$

of linear subspaces of $A$ such that

$$
\begin{aligned}
A^{p} & =\bigoplus_{\substack{1 \leq i \leq p \\
0 \leq q \leq n+1}} A_{i, q} & \text { for all } p, \\
D^{m} & =\bigoplus_{p+q \leq m} A_{p, q} & \text { for all } m, \\
d A_{p, q} & \subset A_{p+q-n-1, n-q+1} & \text { for } p+q>n+1 .
\end{aligned}
$$

Remark 3.2. If $p+q \leq n+1$, then $A_{p, q} \subset D^{n+1}=\operatorname{ker} d$ and, in consequence, $d A_{p, q}=0$. 
Let us introduce the following notation:

$$
\begin{aligned}
V_{p, q} & :=A^{p} \cap D^{p+q}, \\
U_{p, q} & :=A^{p-1} \cap D^{p+q}+A^{p} \cap D^{p+q-1} .
\end{aligned}
$$

Remark 3.3. By Definition 3.1,

$$
V_{p, q}=U_{p, q} \oplus A_{p, q} .
$$

Of course, an arbitrary complement to $U_{p, q}$ in $V_{p, q}$ may not satisfy condition (3.3) of Definition 3.1.

Remark 3.4. From Definition 3.1 and Lemma 2.5 applied to $V=V_{p, q}, U=$ $U_{p, q}, A=A_{p, q}$ and $B=A_{p+q-n-1, n-q+1}$, we obtain that the map $d: A_{p, q} \rightarrow$ $A_{p+q-n-1, n-q+1}$ is an isomorphism for $p+q>n+1$.

The following two observations are essential for applications in the Conley index theory.

Proposition 3.5. The total homology of the $f$-d space $A$ is given by

$$
H(A)=\operatorname{ker} d / \operatorname{Im} d=D^{n+1} / D^{n} \approx \bigoplus_{p+q=n+1} A_{p, q} .
$$

Proposition 3.6. Using the notation $C_{p}=\bigoplus_{q=1}^{n} A_{p, q}$, we have

$$
H\left(A^{p} / A^{p-1}\right) \approx C_{p} .
$$

Proof. By Definition 3.1 and Remark 3.4, we have

- $A^{p}=A^{p-1} \oplus C_{p} \oplus A_{p, 0} \oplus A_{p, n+1}$,

- $d: A_{p, n+1} \rightarrow A_{p, 0}$ is an isomorphism,

- $d C_{p} \subset A^{p-1}$.

Let $\widetilde{d}$ denote the differential on quotients $\widetilde{d}: A^{p} / A^{p-1} \rightarrow A^{p} / A^{p-1}$. We compute

$$
H\left(A^{p} / A^{p-1}\right)=\operatorname{ker} \widetilde{d} / \operatorname{Im} \widetilde{d}=\frac{A^{p-1} \oplus C_{p} \oplus A_{p, 0}}{A^{p-1} \oplus A_{p, 0}} \approx C_{p},
$$

which completes the proof.

Remark 3.7. Similar reasoning shows even more general result. Namely, if $p>q$, then

$$
H\left(A^{p} / A^{q}\right)=\bigoplus_{i=q+1}^{p} C_{i}
$$

Let us introduce the notation for some "blocks" in the spectral splitting. Set

$$
\begin{aligned}
\min & =\min \{r, n+1-p\}, \\
\max & =\max \{n+1-p, n+1-r\}=n+1-\min \{p, r\}
\end{aligned}
$$

for every $p, r=1, \ldots, n$. Here and subsequently, we will use the symbol $A_{p}^{r}$ to denote the direct sum

$$
\bigoplus_{q=\min }^{\max } A_{p, q}
$$


Proposition 3.8. The collection $\left\{A_{p}^{r} ; d: A_{p}^{r} \rightarrow A_{p-r}^{r}\right\}_{p, r=1}^{n}$ is isomorphic to the spectral sequence of the $f$-d space $A$; i.e.,

(1) all the maps $\Phi_{p}^{r}: A_{p}^{r} \rightarrow E_{p}^{r}$ given by

$$
\Phi_{p}^{r}(v):=v+\left(Z_{p-1}^{r-1}+B_{p}^{r-1}\right)
$$

are isomorphisms,

(2) the following diagram commutes

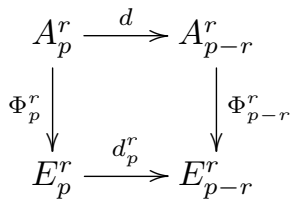

for every $p, r=1, \ldots, n$.

Proof. Since

$$
E_{p}^{r}=\frac{Z_{p}^{r}}{Z_{p-1}^{r-1}+B_{p}^{r-1}}=\frac{A^{p} \cap D^{n+1+p-r}}{A^{p-1} \cap D^{n+p-r}+A^{p} \cap D^{p+r-1}} \approx A_{p}^{r},
$$

it is easy to see that $\Phi_{p}^{r}$ are isomorphisms and the diagram (3.6) commutes for every $p, r=1, \ldots, n$.

Theorem 3.9. There is a spectral splitting for any $f$-d space.

Proof. The construction of a spectral splitting proceeds as follows. If $p+q \leq$ $n+1$, we choose $A_{p, q}$ to be any complement to $U_{p, q}$ in $V_{p, q}$. On the other hand, if $p+q>n+1$, we apply Lemma 2.6 to $V=V_{p, q}, U=U_{p, q}$ and $B=A_{p+q-n-1, n-q+1}$ to get such complement $A_{p, q}$ to $U_{p, q}$ in $V_{p, q}$ that

$$
d A_{p, q} \subset A_{p+q-n-1, n-q+1} .
$$

\section{Spectral splittings and connection matrices}

There is an easy way to obtain connection matrices from spectral splittings as a part of it. Let $C^{p}=\bigoplus_{i=1}^{p} C_{i}$, where $C_{i}=\bigoplus_{q=1}^{n} A_{i, q}$ as before. Then the f-d space $C=\left\{C^{p}\right\}_{p=0}^{n}$ has the following properties:

(1) $C$ is an f-d subspace of $A$, i.e., $C^{p} \subset A^{p}$ for all $p$,

(2) $d C^{p} \subset C^{p-1}$ for all $p$,

(3) the map on homology $i: H(C) \rightarrow H(A)$ induced by the inclusion $C \subset A$ is an isomorphism.

Recall that any f-d space satisfying the above three conditions is called a connection matrix. As we can see a connection matrix is simply a filtration of some "subblocks" of the spectral splitting. Moreover, if we delete from the spectral splitting all direct summands $A_{p, 0}$ and $A_{p, n+1}$ for $p=1, \ldots, n$, we obtain a detailed connection matrix, which is a more refined version of a usual connection matrix (see [4] for definition). 
Remark 4.1. Now assume that all relative homology $H\left(A^{p} / A^{p-1}\right) \approx C_{p}$ are finitely generated. Then all direct summands in detailed connection matrices (as opposed to spectral splittings) are finite-dimensional vector spaces. That easy observation is relevant for applications in the Conley index theory, because it allows us to compare relative homology $H\left(A^{p} / A^{p-1}\right)$ and the total homology $H(A)$ simply by counting bases of the respective vector spaces (see Section 6 for more details).

\section{Spectral graphs}

Definition 5.1. A directed graph $G=(V, E)$ is called a spectral graph for the f-d space $A$ if

(1) $V$ is a set of linearly independent vectors in $A$,

(2) $\operatorname{span}\left\{A^{p} \cap D^{m} \cap V\right\}=A^{p} \cap D^{m}$ for all $p$ and $m$,

(3) each vertex has 0 or 1 edge,

(4) $(v, \widetilde{v}) \in E$ if and only if $d v=\widetilde{v}$,

(5) if $F=\operatorname{span}\{$ the set of vertices with 0 edges\}, then $\operatorname{ker} d=d A \oplus F$ (hence $H(A) \approx F)$.

Remark 5.2. The spectral graph exhibits the following duality:

- edges of the graph give full description of the differential in the f-d space,

- the set of vertices without edges determines the homology of the f-d space $A$.

In other words, the vertices from $C_{p} \approx H\left(A^{p} / A^{p-1}\right)$ which do not contribute to the global homology $H(A)$ are "cancelled" by joining pairs of them with edges.

The following result is a natural consequence of the previous results.

Theorem 5.3. There is a spectral graph for any $f$-d space.

Proof. First, by Theorem 3.9, we get a spectral splitting. Then we choose any bases in the spaces $A_{p, q}$ for $p+q \geq n+1$. Finally bases in $A_{p, q}$ for $p+q<n+1$ are determined by the isomorphisms $d: A_{p+q, n-q+1} \rightarrow A_{p, q}$.

Remark 5.4. The proof of Theorem 5.3 shows how to obtain a spectral graph from a spectral splitting. However, the reverse reconstruction is also possible. Namely, condition (2) of Definition 5.1 guarantees that using the formula

$$
A_{p, q}=\operatorname{span}\left\{\left(V_{p, q} \cap V\right) \backslash\left(U_{p, q} \cap V\right)\right\},
$$

we can recover a spectral splitting from a spectral graph.

The vertices of a spectral graph may be labeled as follows. Each vertex $v$ is labeled by an integer $l(v)=p:=\max \left\{i \mid v \in A^{i}\right\}$. We use $v_{p}$ to denote a vertex labeled by $p$. Of course, two different vertices may have the same label. Moreover, let $W=\{v \in V \mid d v \in V$ and $l(v)=l(d v)\}$. A full subgraph of a spectral graph spanned by the vertices $V \backslash(W \cup d W)$ will be called a connection graph. 


\section{Morse decompositions}

Spectral splittings and graphs may be used for studying the structure of Morse decompositions.

Let $X$ be a compact metric space. Assume we have a flow on $X$. Recall that a collection $\left\{M_{i}\right\}_{1}^{n}$ of mutually disjoint compact invariant subsets of $X$ is called a Morse decomposition if for every $x \in X \backslash \bigcup_{i=1}^{n} M_{i}$ there are indices $i<j$ such that $\omega^{+}(x) \subset M_{i}$ and $\omega^{-}(x) \subset M_{j}$. The sets $M_{i}$ are called Morse sets. Moreover, generalized Morse sets for $i \leq j$ are defined as

$$
M_{j i}:=\left\{x \in X \mid \omega^{+}(x) \cup \omega^{-}(x) \subset \bigcup_{k=i}^{j} M_{k}\right\} .
$$

Spectral splittings and graphs may be naturally related to a filtration of index pairs associated with the Morse decomposition. Recall that an increasing filtration of compact sets $\left\{N^{i}\right\}_{0}^{n}$ is called an index filtration if

(1) $N^{0}=\emptyset$ and $N^{n}=X$,

(2) $\left(N^{j}, N^{i-1}\right)$ is an index pair for $M_{j i}$.

Such filtrations, even regular, exist (see $[11,14]$ for more details). Recall that if the index filtration is regular, then the Conley index of each Morse set $M_{j i}$ is isomorphic to the singular homology of the pair $\left(N^{j}, N^{i-1}\right)$.

Let $\left\{N^{i}\right\}_{0}^{n}$ be an index filtration for the Morse decomposition $\left\{M_{i}\right\}_{1}^{n}$. Let $C\left(N^{k}\right)$ be the vector space of singular chains in $N^{k}$ and let $i: C\left(N^{k}\right) \rightarrow$ $C(N)$ be a homomorphism induced by the inclusion $N^{k} \subset N$. It is evident that a filtration

$$
\left\{i\left(C\left(N^{k}\right)\right)\right\}_{0}^{n}
$$

equipped with the boundary map on singular chains, is an f-d space. A spectral splitting (graph) for the Morse decomposition $\left\{M_{i}\right\}_{1}^{n}$ is simply a spectral splitting (graph) for the above f-d space.

Repeating the reasoning from the proof of [1, Theorem 7.2] we can easily show the following result.

Proposition 6.1. Any two spectral splittings (spectral graphs) for a given Morse decomposition are isomorphic; i.e., they are independent (up to isomorphism) of the choice of an index filtration.

In our next theorem we will make use of the following definition.

Definition 6.2. We say that two Morse sets $M_{p}$ and $M_{q}$ are linked if there are a sequence of Morse sets $M_{i_{1}}, \ldots, M_{i_{k}}$ and a sequence of orbits $\gamma_{1}, \ldots, \gamma_{k-1}$ such that

- $M_{i_{1}}=M_{p}$ and $M_{i_{k}}=M_{q}$,

- $M_{i_{m}} \cap \operatorname{cl} \gamma_{m} \neq \emptyset$ and $M_{i_{m+1}} \cap \operatorname{cl} \gamma_{m} \neq \emptyset$ for $m=1, \ldots, k-1$.

Our last result shows how to use spectral graphs in analyzing the structure of the Morse decomposition. It is worth pointing out that even an easy comparison of local Conley indices of Morse sets with the total homology of the whole space by means of the spectral graph may provide information on how different Morse sets are linked. 
Theorem 6.3. Let $G=(V, E)$ be a spectral graph for the Morse decomposition $\left\{M_{j}\right\}_{1}^{n}$. If $n \geq p>s \geq 1$ and $\left(v_{p}, v_{s}\right) \in E$, then the Morse sets $M_{p}$ and $M_{s}$ are linked.

Proof. Let $n \geq p>s \geq 1$ and $\left(v_{p}, v_{s}\right) \in E$. Using the notation from Remark 5.4 we see that

$$
v_{p} \in A_{p, n+s-p+1} .
$$

Suppose now that the Morse sets $M_{p}$ and $M_{s}$ are not linked. Hence there exists an index filtration $\left\{N^{j}\right\}_{0}^{n}$ for the Morse decomposition $\left\{M_{j}\right\}_{1}^{n}$ such that for some $k(p>k \geq s)$ both sets $N^{p} \backslash N^{k}$ and $N^{k}$ are closed in $X$. Denoting

$$
A^{j}:=i\left(C\left(N^{j}\right)\right) \text { and } B:=i\left(C\left(N^{p} \backslash N^{k}\right)\right),
$$

we obtain $A^{p}=A^{k} \oplus B$. Hence $v_{p}=v^{\prime}+v^{\prime \prime}$, where $v^{\prime} \in A^{k}$ and $v^{\prime \prime} \in B$. This gives $v_{s}=d v_{p}=d v^{\prime}+d v^{\prime \prime}$ and consequently

$$
A^{k} \ni v_{s}-d v^{\prime}=d v^{\prime \prime} \in B,
$$

because the differential on singular chains operates component by component. We thus get $v^{\prime \prime} \in \operatorname{ker} d=D^{n+1}$ and so

$$
v_{p}=v^{\prime}+v^{\prime \prime} \in A^{k} \cap D^{n+s+1}+A^{p} \cap D^{n+1} \subset U_{p, n+s-p+1},
$$

contrary to $(6.1)$.

We end this section with two simple examples illustrating the computation of connection graphs. The second example is adapted from [12].

Example (A gradient flow on a deformed 2-sphere). Consider the gradient flow of the height function on a manifold $M$ diffeomorphic to $S^{2}$ with phase portrait as in Figure 1. Observe that two minima (1 and 2), two maxima (4 and 5) and a "circle" consisting of two saddle points and two connecting orbits between them (3) form a Morse decomposition of $M$. Moreover, the respective sublevel sets on $M$ form a natural index filtration. It will be convenient to represent a connection graph for the Morse decomposition in a tabular form. The columns are labeled by Morse sets and the rows correspond

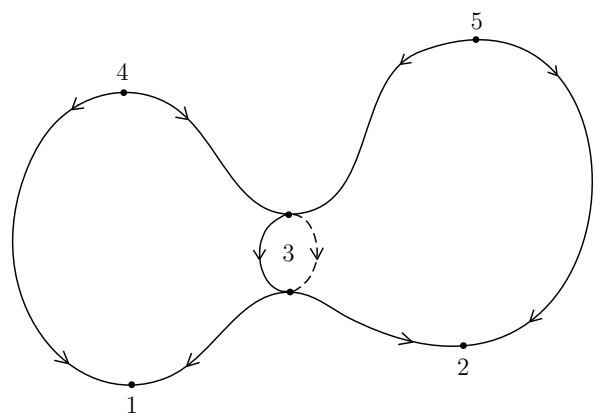

Figure 1. A gradient flow on a deformed 2-sphere. 
to the gradation on homology. Of course, dots (arrows) represent vertices (edges).

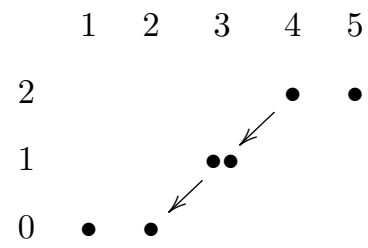

As we have mentioned before, the connection graph shows the relationship between the Conley indices of Morse sets (columns) and the homology groups of the manifold $M$ (vertices without edges). Besides, the edges describe the action of the differential of the respective $\mathrm{f}-\mathrm{d}$ space.

Example (A flow on the 2-sphere). Consider the local flow in $\mathbb{R}^{2}$ with phase portrait as in Figure 2 (see [12] for more details). If we consider the flow as a flow on the 2 -sphere, then the point at infinity becomes an attractor. Observe that three repelling rest points $(2,6,7)$, two saddle rest points $(4,5)$, one attracting rest point (3) and one attracting periodic orbit (1) form a Morse decomposition of $S^{2}$. We can easily construct an index filtration for this Morse

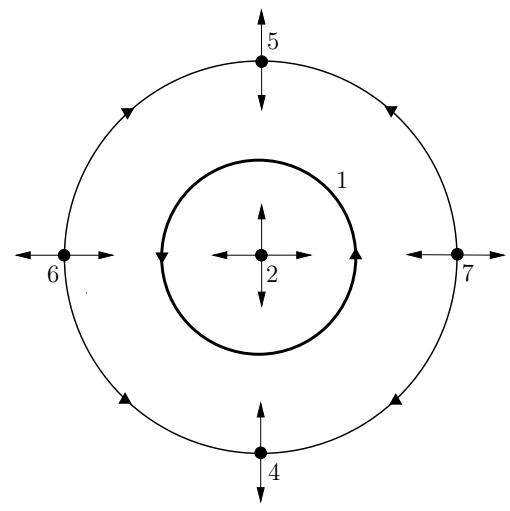

Figure 2. A flow on the 2-sphere.

decomposition, which uniquely determines a connection graph (see below).

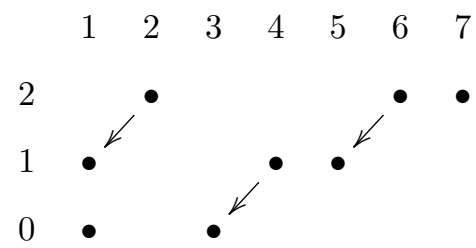

It may be worth pointing out that the phenomenon of nonuniqueness of connection matrices (graphs) cannot be observed here (see Proposition 6.1). 


\section{Acknowledgment}

The author wants to express his thanks to the referee for several helpful comments concerning the paper.

\section{References}

[1] P. Bartłomiejczyk, The Conley index and spectral sequences. Topol. Methods Nonlinear Anal. 25 (2005), 195-203.

[2] P. Bartłomiejczyk, Connection graphs. Fund. Math. 192 (2006), 93-110.

[3] P. Bartłomiejczyk, Simple connection matrices. Ann. Polon. Math. 90 (2007), $77-87$.

[4] P. Bartłomiejczyk, Spectral sequences and detailed connection matrices. Topol. Methods Nonlinear Anal. 34 (2009), 187-200.

[5] C. Conley, Isolated Invariant Sets and the Morse Index. CBMS Reg. Conf. Ser. Math. 38, Amer. Math. Soc., Providence, RI, 1978.

[6] C. Conley and E. Zehnder, Morse-type index theory for flows and periodic solutions for Hamiltonian equations. Comm. Pure Appl. Math. 37 (1984), 207253.

[7] O. Cornea, K. A. de Rezende and M. R. Silveira, Spectral sequences in Conley's theory. Ergodic Theory Dynam. Systems 30 (2010), 1009-1054.

[8] B. Fiedler and K. Mischaikow, Dynamics of bifurcations for variational problems with $O(3)$ equivariance: A Conley index approach. Arch. Ration. Mech. Anal. 119 (1992), 145-196.

[9] R. Franzosa, The connection matrix theory for Morse decompositions. Trans. Amer. Math. Soc. 311 (1989), 561-592.

[10] K. Mischaikow and M. Mrozek, The Conley index theory. In: Handbook of Dynamical Systems II: Towards Applications, Elsevier, Singapore, 2002, 393460 .

[11] M. R. Razvan, On Conley's fundamental theorem of dynamical systems. Int. J. Math. Math. Sci. 2004 (2004), 1397-1401.

[12] J. Reineck, The connection matrix in Morse-Smale flows. Trans. Amer. Math. Soc. 322 (1990), 523-545.

[13] J. W. Robbin and D. Salamon, Lyapunov maps, simplicial complexes and the Stone functor. Ergodic Theory Dynam. Systems 12 (1992), 153-183.

[14] D. Salamon, Connected simple systems and the Conley index of isolated invariant sets. Trans. Amer. Math. Soc. 291 (1985), 1-41.

[15] E. C. Zeeman, On the filtered differential group. Ann. of Math. (2) 66 (1957), $557-585$.

Piotr Bartłomiejczyk

Institute of Mathematics

University of Gdańsk

Wita Stwosza 57

80-952 Gdańsk

Poland

e-mail: pb@mat.ug.edu.pl

Open Access This article is distributed under the terms of the Creative Commons Attribution License which permits any use, distribution, and reproduction in any medium, provided the original author(s) and the source are credited. 